\title{
A fuzzy based vertical handover network selection scheme for device-to-device communication
}

\author{
Meenakshi Subramani, Vinoth Babu Kumaravelu \\ School of Electronics Engineering, Vellore Institute of Technology, Vellore, Tamil Nadu, India
}

\begin{tabular}{l}
\hline Article Info \\
\hline Article history: \\
Received Apr 1, 2019 \\
Revised Jun 17, 2019 \\
Accepted Jul 7, 2019 \\
\hline
\end{tabular}

Keywords:

Analytic hierarchy process (AHP)

Device-to-Device (D2D)

communication

Fuzzy logic

Handover decision delay

Simple additive weighting

(SAW)

Vertical Handover (VHO)

\begin{abstract}
One of the most attractive and challenging areas in the upcoming next-generation $5 \mathrm{G}$ wireless network is the vertical handover (VHO). Recently, many of the heterogeneous wireless communication technologies are introduced to satisfy the demands of users in all situations. Due to the deployment of heterogeneous networks, the users can access the internet anywhere, anytime through different wireless networks. To obtain seamless service and service continuity, the device should be handed over to the best wireless networks. Here, a half handover scheme for Device-to-Device (D2D) communication is implemented for the selection of the best network. The target network selection for vertical handover can be handled using multiple attribute decision making (MADM) methods. An intelligent and fast vertical handover decision is much needed, which should be reliable even for random and uncertain environments. Fuzzy logic is proved to be effective in handling imprecise data. Hence, in this work, the impact of combining fuzzy with the conventional MADM scheme, simple additive weighting (SAW) is analyzed and the hybrid scheme is compared with the conventional MADM schemes like SAW, Techniques for order preference by similarity to ideal solution (TOPSIS), VlseKriterijumska optimizacija I Kompromisno Resenje (VIKOR) in terms of handover decision delay. Since, the numbers of handovers executed are low, the handover decision delay performance of the proposed scheme is superior than the considered classical MADM schemes.
\end{abstract}

\section{Corresponding Author:}

Vinoth Babu Kumaravelu, School of Electronics Engineering, Vellore Institute of Technology, Vellore, Tamil Nadu, India. Email: vinothbab@gmail.com

\section{INTRODUCTION}

Due to increased demands of the users, $5 \mathrm{G}$ will overcome the shortages of $4 \mathrm{G}$ communication, which is expected to offer higher data rate, increased voice quality calls, improved spectrum efficiency, reduced latency, etc. to the end users [1]. 5G networks will support the emerging and existing technologies, which integrates newer solutions to obtain the increasing demand for higher data rate. One of the important features of $5 \mathrm{G}$ is D2D communication [2]. In D2D, two devices in close proximity communicate directly rather than communicating through the evolved node base station (eNB) or the core network.

Due to its direct link communication, it offloads data traffic and improves spectral efficiency. It also reduces power consumption and latency. In $5 \mathrm{G}$, the concept of small cells is very popular, where the coverage range of access points are reduced [3]. Due to the mobility nature, the devices undergoing D2D communication requires frequent handovers [2]. The selection of the target network is to ensure proper communication and seamless service between the devices, which acts as a basic requirement in many practical applications. To achieve always best connection $(\mathrm{ABC})$ and to meet the good quality of service (QoS), the device has to 
switch to different networks [4]. The process of switching to different networks is called VHO and the process of switching between the same networks is called horizontal handover [2]. The interesting and challenging research area in the wireless environment is to select the best network from several candidate networks. When one of the devices undergoing D2D communication moves away from the other device, the link quality between them becomes very poor. This leads to poor QoS and connection breakdown. Hence, one of the devices is handed over to the other network. After the handover, these devices may continue their communication through cellular links. This type of handover is termed as half handover. A sample D2D scenario requiring handover is shown in Figure 1a. D1 and D2 marked in Figure 1a represent the devices undergoing D2D communication. The process of half handover is illustrated in Figure 1b. In some cases, both the devices undergoing D2D communication may move towards the neighboring network. For seamless service continuity, both the devices are jointly handed over to the best neighboring network. This process is termed as joint handover [2], which is illustrated in Figure 1c.

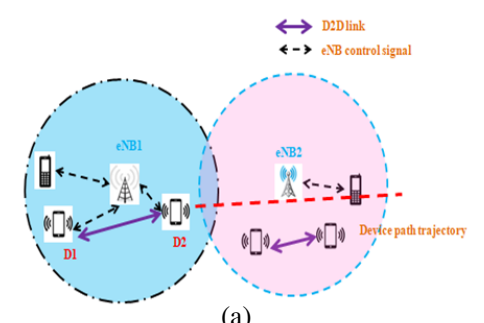

(a)

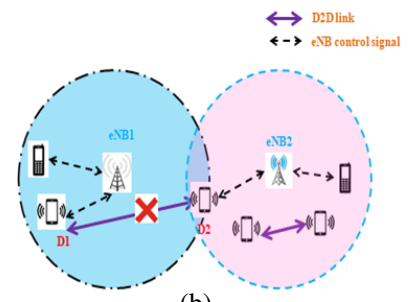

(b)

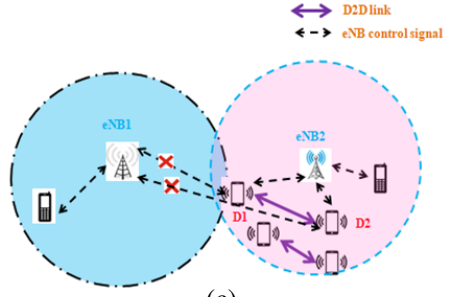

(c)

Figure 1. (a) A scenario for the device in D2D communication requiring handover, (b) After half handover, (c) After joint handover

Target network selection is an important task to achieve a seamless connection and QoS in VHO environment. The network gathers the parameters from every candidate network and ranks them to choose the best target network. Most of the conventional handover schemes make use of received signal strength (RSS) to select the target network $[4,5]$. This introduces a ping-pong effect, which leads to decreased throughput, increased latency and dropping rate. Many of the recent approaches make use of various parameters to decide the handover and the target network. These include a signal to noise ratio (SNR), achievable bit rate, bit error rate (BER), outage probability, cost, security, power consumption, available bandwidth, etc. [4].

Due to its simplicity in operation, MADM algorithms are widely used in VHO decision making and target network selection. In literature, there exist many compensatory and non-compensatory MADM algorithms. Most of the network selection algorithms proposed are based on the compensatory method. The compensatory algorithms combine multiple criteria to find the best network, whereas non-compensatory algorithms combine multiple criteria to find the acceptable network, which satisfies the minimum requirements. SAW [6], TOPSIS [7], VIKOR [8] algorithms come under compensatory category. These are popular for lower computational complexity and improved accuracy in decision making.

Fuzzy logic models complex systems fairly without any bias, which is not in the case of the AHP process $[9,10]$. Fuzzy logic is capable of processing a large number of inputs and makes a soft decision. It efficiently handles the imprecise data and represents it in an innate form [3]. Most of the conventional VHO decision-making algorithms are based on RSS, which fluctuates based on various parameters like distance, mobility, speed and shadowing factor, etc. The imprecise input may cause inaccurate decision in deciding the handover. This leads to over or under-utilization of network resources. Fuzzy logic is proved to be efficient in handling the data related to radio, QoS and user preferences [4].

\section{THE PROPOSED METHOD}

In this work, fuzzy logic is combined with the conventional AHP and SAW methods to simultaneously process a large number of inputs and to effectively handle the imprecise data related to handover decision making and target network selection. Based on the input criteria, Fuzzy AHP is used to calculate the weights of each criteria. These weights and the available candidate networks are given as the input for the Fuzzy SAW scheme. The network with the highest rank is chosen as the target network to handover. The proposed method for multi-criteria network selection block diagram is shown in Figure 2. 
The rest of the work is arranged as follows: An introduction to fuzzy theory, Fuzzy AHP and Fuzzy SAW are explained in section 3. The results are discussed in section 4 and the paper is concluded in section 5.

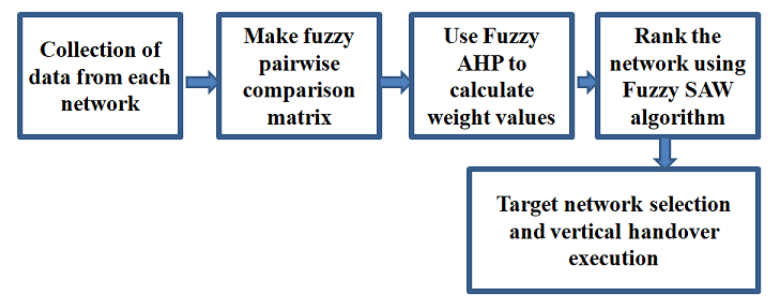

Figure 2. Block diagram of Fuzzy AHP-Fuzzy SAW MADM network selection

\section{RESEARCH METHOD}

\subsection{Fuzzy theory}

Fuzzy set theory denotes the ambiguous data in an innate form [3]. Due to the lower computational complexity and simpler mathematical implementation, triangular fuzzy number (TFN) is widely preferred for the applications related to wireless communication. It is also proved that the complexities related to handling imprecise and uncertainty information used for network selection is minimized with TFN. Hence, in this work, TFN is utilized to establish ambiguity metrics. TFN is one of the important classification of fuzzy number with its membership function defined by $(x, y, z)$, three crisp numbers as $(x \leq y \leq z)$, where $x$ is the lower limit, $y$ is the modal value and $z$ is the upper limit. When $x=y=z$, then the fuzzy number will become a real number. A fuzzy set $\widetilde{G}$ in a universe of discourse $A$ is represented by a membership function $\mu_{\widetilde{G}}(a)$, which is related to each element $a$ in $A$ and the real number with an interval $[0,1]$. The $\mu_{\widetilde{G}}(a)$ function value is referred as the membership grade of $a$ in $\widetilde{G}$. The fuzzy number $\widetilde{G}$ on $\mathbb{R}$ to be TFN, when the membership function, $\mu_{\widetilde{G}}(a): \mathbb{R} \rightarrow[0,1]$. TFN can be defined as

$$
\mu_{\widetilde{G}}(a)= \begin{cases}\frac{(a-x)}{(y-x)} & , x \leq a \leq y \\ \frac{(z-a)}{(z-y)} & , y \leq a \leq z \\ 0, \text { otherwise }\end{cases}
$$

\subsection{Fuzzy AHP}

AHP is mainly used for decision-making in multi-criteria problems, which is initiated with a measurement of ratio scales [11]. AHP use comparison and pre-set option selection, which depends on the pairwise comparisons between the options and criteria.

Here, a qualitative judgment is used for pairwise comparison. Fuzzy AHP is very easy and simple to analyze in making decisions. Fuzzy AHP causes the significance of fuzzy, which occurs in the same row with two combination items. The fuzzy extension is needed because the basic AHP failed to address the main issue of handling the high degree of imprecise in subjective personal judgments and its preferences. Fuzzy AHP is applicable to tackle the problem at hand by considering the structure of multi-criteria and vagueness in a real-time environment, which improves the consistency in the network selection. Fuzzy AHP uses a 9-point fundamental scale, which is expressed in terms of TFN to indicate the relative performance between the pairwise decision factors. The TFN values are represented in Table 1.

Table 1. 9-point fundamental fuzzy scale using TFN [12]

\begin{tabular}{llrr}
\hline Fuzzy scale & & & \\
intensity of importance & Linguistic scale description & TFN & Reciprocal of TFN \\
\hline$\widetilde{1}$ & Equal importance & $(1,1,1)$ & $(1,1,1)$ \\
$\widetilde{2}$ & Moderately equal important & $(1,2,3)$ & $(1 / 3,1 / 2,1)$ \\
$\widetilde{3}$ & Moderately important & $(1,3,5)$ & $(1 / 5,1 / 3,1)$ \\
$\widetilde{4}$ & Moderately strongly important & $(2,4,6)$ & $(1 / 6,1 / 4,1 / 2)$ \\
$\widetilde{5}$ & Strongly important & $(3,5,7)$ & $(1 / 7,1 / 5,1 / 3)$ \\
$\widetilde{6}$ & Strongly very important & $(4,6,8)$ & $(1 / 8,1 / 6,1 / 4)$ \\
$\widetilde{7}$ & Very strongly important & $(5,7,9)$ & $(1 / 9,1 / 7,1 / 5)$ \\
$\widetilde{8}$ & Very strongly extremely important & $(6,8,9)$ & $(1 / 9,1 / 8,1 / 6)$ \\
$\widetilde{9}$ & Extremely important & $(7,9,9)$ & $(1 / 9,1 / 9,1 / 7)$ \\
\hline
\end{tabular}


The Fuzzy AHP has the following steps:

Step 1: Based on the objectives, select the suitable criteria.

Step 2: Construct a pairwise comparison matrix $\widetilde{R}$ based on each criteria. The elements $\widetilde{r_{i j}}$ in $\widetilde{R}$ represents the pairwise comparison of criteria $i$ with $j$.

$$
\widetilde{R}=\left[\widetilde{r_{i j}}\right]_{n \times n}=\left[\begin{array}{lll}
\widetilde{r_{11}} & \widetilde{r_{12}} & \widetilde{r_{13}} \cdots \widetilde{r_{1 n}} \\
\widetilde{r_{21}} & \widetilde{r_{22}} & \widetilde{r_{23}} \cdots r_{2 n} \\
\vdots & \vdots & \vdots \\
\widetilde{r_{n 1}} & \widetilde{r_{n 2}} & \widetilde{r_{n 3}} \cdots \widetilde{r_{n n}}
\end{array}\right]
$$

where $n$ denotes the number of criteria decision compared and $i=j=1,2, \ldots . n . \widetilde{r_{i j}}$ represents the relative strength of two elements based on TFN.

$$
\widetilde{r_{j i}}=\left[\widetilde{r_{i j}}\right]^{-1}=\left(x_{i j}, y_{i j}, z_{i j}\right)^{-1}=\left(\frac{1}{z_{i j}}, \frac{1}{y_{i j}}, \frac{1}{x_{i j}}\right)
$$

Step 3: The Fuzzy AHP comparison matrix is represented with TFN as

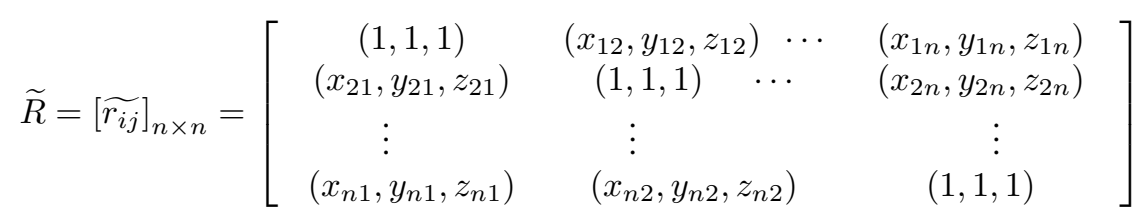

Step 4: The fuzzy geometric mean and fuzzy weight for each criteria is calculated as [13, 14]

$$
\begin{aligned}
\widetilde{l_{i}} & =\left(\widetilde{r_{i 1}} \otimes \widetilde{r_{i 2}} \otimes \ldots . \otimes \widetilde{r_{i n}}\right)^{1 / n} \\
\widetilde{w_{i}} & =\widetilde{l_{i}} \otimes\left(\widetilde{l_{1}} \oplus l_{2} \oplus \cdots \oplus l_{n}\right)^{-1}
\end{aligned}
$$

The sign $\otimes$ indicates fuzzy multiplication and sign $\oplus$ indicates fuzzy addition. $\widetilde{r_{i n}}$ is the fuzzy comparison value of $i$ th criteria to $n$th criteria, $\widetilde{l}_{i}$ is the geometric mean of fuzzy comparison value of $i$ th criteria to each criteria, $\widetilde{w_{i}}$ is the fuzzy weight of $i$ th criteria.

Step 5: In order to check the inconsistency in the pairwise comparison matrix, consistency index (CI) is introduced to obtain the consistency ratio $(\mathrm{CR})$. $\mathrm{CI}$ is given as

$$
C I=\frac{\lambda_{\max }-n}{n-1}
$$

where

$$
\lambda_{\max }=\frac{\widetilde{R} \cdot \widetilde{w}}{\widetilde{w}}
$$

where $\lambda_{\max }$ is the largest Eigen value of the comparison matrix $\widetilde{R}, n$ represents the number of criteria and $\widetilde{w}$ is the weight value calculated to obtain Eigen vector. Based on the CI, the value of CR is calculated as

$$
C R=\frac{C I}{R I}
$$

where RI is the random consistency index as shown in Table 2. Various authors measured RI values for a different number of criteria. This is tabulated in [15]. In [15], the authors estimated RI for each number of criteria using 100,000 matrices. They have generated random matrices with uniform distribution. Then, CIs are calculated for each matrix. The RI for each number of criteria is obtained by taking mean of these CI values. It is proved that the RI value calculated by [16] is efficient than the other methods. Hence, in this work, we have used the same table, which was listed in [16]. If the estimated CR value is less than 0.1 , then the pairwise construction is acceptable, otherwise, the matrix has to be revised [17].

Table 2. Random consistency value [16]

\begin{tabular}{lcccccccccc}
\hline Criteria & 1 & 2 & 3 & 4 & 5 & 6 & 7 & 8 & 9 & 10 \\
\hline RI & 0 & 0 & 0.58 & 0.9 & 1.12 & 1.24 & 1.32 & 1.41 & 1.45 & 1.49 \\
\hline
\end{tabular}




\subsection{Fuzzy SAW}

Because of its simplicity, Fuzzy SAW is widely used in the MADM algorithms[18, 4]. Fuzzy SAW requires the normalizing procedure for the decision matrix into a scale, which is compared with every network rating. The main idea of Fuzzy SAW method is to detect the weighted sum of performance rating of every network on each criteria. The steps are as follows:

Step 1: Weight calculation Obtain the weight value for each criteria from Fuzzy AHP method.

Step 2: Fuzzy decision matrix Formulate the decision matrix $\widetilde{F}$ and select the suitable linguistic variables with respect to different networks and criteria. A $q \times n$ fuzzy decision matrix is formulated with the ratings of each network with each criteria. The entries are TFN instead of crisp values.

$$
\widetilde{F}=\begin{aligned}
& N w_{1} \\
& N w_{2} \\
& N w_{3} \\
& \vdots \\
& N w_{q}
\end{aligned}\left[\begin{array}{ccccc}
\widetilde{b_{11}} & \widetilde{C_{12}} & \widetilde{b_{3}} & \cdots & C_{n} \\
\widetilde{b_{21}} & \widetilde{b_{22}} & \widetilde{b_{23}} & \cdots & \widetilde{b_{1 n}} \\
\widetilde{b_{31}} & \widetilde{b_{32}} & \widetilde{b_{33}} & \cdots & \widetilde{b_{3 n}} \\
\vdots & \vdots & \vdots & \cdots & \vdots \\
\widetilde{b_{q 1}} & \widetilde{b_{q 2}} & \widetilde{b_{q 3}} & \cdots & \widetilde{b_{q n}}
\end{array}\right]
$$

where $N w_{1}, N w_{2}, N w_{3}$,

$N w_{q}$ are the possible networks, $C_{1}, C_{2}, C_{3}$, $C_{n}$ are the criteria. The element $\widetilde{b_{i j}}$ is the fuzzy decision matrix, which indicates the performance rating of each network $N w_{i}$ with respect to criteria $C_{j}$, where $i=1,2,3, \ldots . . q$ and $j=1,2,3, \ldots . . n$ respectively.

Step 3: Fuzzy normalization weight value The fuzzy decision matrix $\widetilde{F}$ depends on the linguistic variables and the corresponding TFN. Each entry of $\widetilde{F}$ is TFN, which corresponds to 3 values, i.e, $\widetilde{b_{i j x}}, \widetilde{b_{i j y}}, \widetilde{b_{i j z}}$. The normalized fuzzy decision matrix $\widetilde{g_{i j}}$ is given by

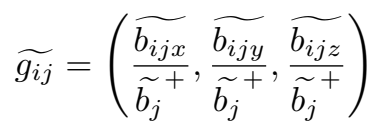

For benefit criteria, the maximum of 3rd value of TFN in the fuzzy decision matrix $\widetilde{F}$ is identified using

$$
{\widetilde{b_{j}}}^{+}=\max _{i} \widetilde{b_{i j z}} \text {, where } j \in B
$$

where $B$ is the set of benefit criteria, which is always expected to be maximum. All the elements of $\widetilde{F}$ are normalized using ${\widetilde{b_{j}}}^{+}$.. Alternatively, cost criteria can also be used. The normalized fuzzy decision matrix $\widetilde{g_{i j}}$ for cost criteria is given by

$$
\widetilde{g_{i j}}=\left(\frac{\widetilde{c_{j}}}{\widetilde{\widetilde{b_{i j z}}}}, \frac{\widetilde{c_{j}}}{\widetilde{b_{i j y}}}, \frac{\widetilde{c_{j}}}{\widetilde{b_{i j x}}}\right)
$$

For cost criteria, the minimum of the 1 st value of TFN in the fuzzy decision matrix $\widetilde{F}$ is identified using

$$
\widetilde{c_{j}}-\min _{i} \widetilde{b_{i j x}} \text {, where } j \in C
$$

where $C$ is the set of cost criteria, which is always expected to be minimum. All the elements of $\widetilde{F}$ are normalized using $\widetilde{c_{j}}{ }^{-}$.

For benefit criteria, the highly acceptable value is considered as the best value. For example, RSS and data rate. For cost criteria, the lower acceptable value is considered as the best value.

Step 4: Weight normalization value In this step, a weight normalized decision matrix $\left(\widetilde{M_{i j}}\right)$ is formulated by multiplying each element $\widetilde{g_{i j}}$ of normalized fuzzy decision matrix with the weights $\widetilde{w}$ obtained through Fuzzy AHP in step 4 of section 3 as

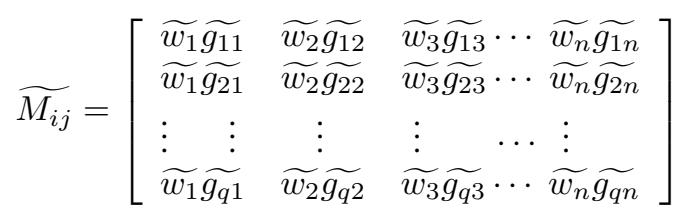




\section{Step 5: Network ranking}

Fuzzy SAW algorithm evaluates all the networks and makes a decision to perform handover. The overall rank of $i$ th network is evaluated using

$$
D_{S A W_{i}}=\frac{1}{n} \sum_{j=1}^{n} \widetilde{g_{i j}} \cdot \widetilde{w_{j}}
$$

\section{RESULTS AND DISCUSSION}

MATLAB 2017a tool is used for simulation. The considered simulation parameters are listed in Table 3. Figure 3 shows the handover decision delay versus a number of inputs. The simulations are carried out for streaming applications. During the vertical handover decision, the handover decision delay is considered as one of the important parameters. The delay in the handover decision leads to QoS degradation. If the handover decision is done too early, it leads to unnecessary handover. The goal is to measure the handover decision delay for the increased number of inputs. In many real-world problems, decision-makers may not be sure about their preferences. This leads to uncertainty. The inclusion of fuzzy logic effectively handles various decision-making problems. In Fuzzy AHP, the pairwise comparison matrix is formulated with the help of TFN. This avoids ambiguities in finding the weights. These weights are used in fuzzy SAW for ranking and selecting the target networks. The decision makers also use the fuzzy logic in formulating the decision matrix which compares alternatives with criteria. Since, the uncertainties are handled effectively and fast, the handover decision time during the selection of the target network is very low for the Fuzzy AHP-Fuzzy SAW scheme.

Table 3. Simulation parameters

\begin{tabular}{lc}
\hline Parameters & Values \\
\hline Networks & Wi-Fi / WiMAX / LTE-A \\
Cell radius $(\mathrm{km})$ & Wi-Fi: 0.25; WiMAX: 10; LTE-A: 3 \\
Transmit Power $(\mathrm{dBm})$ & Wi-Fi: 13; WiMAX: 47; LTE-A: 46 \\
Bandwidth (MHz) & Wi-Fi: 20; WiMAX: 40; LTE-A: 100 \\
Path loss model for Wi-Fi & $P L(d B)^{W i-F i}=34.48+32.79 \log _{10} d(\mathrm{~m})[2]$ \\
Path loss model for WiMAX & $P L(d B)^{W i M A X}=130.62+37.6 \log _{10} d(\mathrm{~km})[2]$ \\
Path loss model for LTE-A & $P L(d B)^{L T E-A}=103.8+20.9 \log _{10} d(\mathrm{~km})[2]$ \\
\hline
\end{tabular}

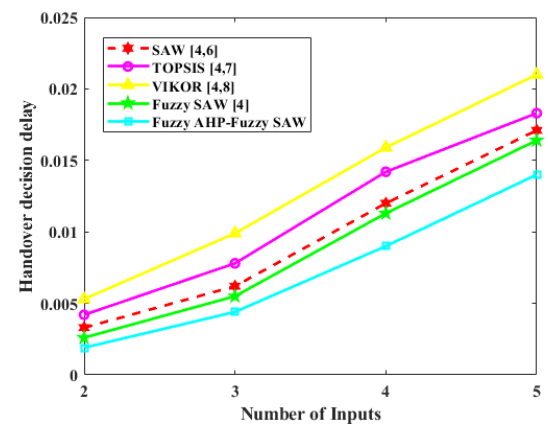

Figure 3. Handover decision delay versus number of inputs comparison of various MADM schemes

For three criteria, Fuzzy AHP-Fuzzy SAW offers 29.03 \%, $43.59 \%, 55.55 \%, 20 \%$, reduction in handover decision delay over the conventional SAW [4, 6], TOPSIS [4, 7], VIKOR [4, 8] and Fuzzy SAW [4] schemes. For any number of increased inputs, Fuzzy AHP-Fuzzy SAW handover decision time will be lesser. Hence, Fuzzy AHP-Fuzzy SAW selects the best target network to perform an efficient, fast and seamless handover.

\section{CONCLUSION}

Since D2D communication mostly happens for a smaller duration, the handover decision delay should be much smaller for a seamless connection. Most of the conventional MADM schemes are not fast and reliable. They also fail when handling the imprecise data. Hence, in this work, the concept of fuzzy is combined with 
the conventional SAW and the hybrid scheme Fuzzy AHP-Fuzzy SAW is proved to offer improved handover decision delay performance over SAW, TOPSIS, VIKOR, Fuzzy SAW schemes. Due to lower handover decision delay, the packet drop ratio and service interruption are greatly reduced for the proposed scheme. This increases the average data rate experienced by each device. This also minimizes the latency of the proposed scheme. This simple work can be extended for other criteria, networks and MADM algorithms. As a future study, these schemes can also be combined and tested with neuro-fuzzy based algorithms.

\section{REFERENCES}

[1] S.T. Shah, et al., "Device-to-device communications: A contemporary survey", Wireless Personal Communications, 98, No.1, pp.1247-1284, 2018.

[2] M. Subramani, and V.B. Kumaravelu, "A Quality-Aware Fuzzy-Logic-Based Vertical Handover Decision Algorithm for Device-to-Device Communication”, Arabian Journal for Science and Engineering, pp.1-13, 2018.

[3] A. Murugadass, and A. Pachiyappan, "Fuzzy Logic Based Coverage and Cost-Effective Placement of Serving Nodes for 4G and Beyond Cellular Networks", Wireless Communications and Mobile Computing, 2017.

[4] A.B Zineb, et al., "An enhanced vertical handover based on fuzzy inference MADM approach for heterogeneous networks", Arabian Journal for Science and Engineering, 42, No.8, pp.3263-3274, 2017.

[5] T. Thumthawatworn, et al., "Adaptive Multi-fuzzy Engines for Handover Decision in Heterogeneous Wireless Networks", Wireless Personal Communications, 93, No.4, pp.1005-1026, 2017.

[6] P. Bagga, A. Joshi and R. Hans, "QoS based Web Service Selection and Multi-Criteria Decision Making Methods", International Journal of Interactive Multimedia and Artificial Intelligence, Vol. 5, No. 4, 2019.

[7] H. Lam Weng, S. Lam Weng and F. Liew Kah, "Performance analysis on telecommunication companies in Malaysia with TOPSIS model", Indonesian Journal of Electrical Engineering and Computer Science, Vol. 13, No. 2, PP. 744-751, 2019.

[8] S.A.A. Alrababah, et al., "Comparative analysis of MCDM methods for product aspect ranking: TOPSIS and VIKOR", IEEE International Conference on Information and Communication Systems, pp. 76-81, 2017.

[9] N. Hamidy, H. Alipur, S.N.H. Nasab, A. Yazdani and S. Shojaei, "Spatial evaluation of appropriate areas to collect runoff using Analytic Hierarchy Process (AHP) and Geographical Information System (GIS)(case study: the catchment "Kasef" in Bardaskan)" Modeling Earth Systems and Environment, 2, No. 4, pp.1-11, 2016.

[10] S. Shojaei, H. Alipur, A.H.H. Ardakani, S.N.H. Nasab and H. Khosravi, "Locating Astragalus hypsogeton Bunge appropriate site using AHP and GIS", Spatial Information Research, 26, No. 2, pp.223-231, 2018.

[11] S.A. Mohammed, "Solving Course Selection Problem by a Combination of Correlation Analysis and Analytic Hierarchy Process", International Journal of Electrical and Computer Engineering, Vol. 7, No. 6, pp. 3536-3551, 2017.

[12] B.M. Elomda, et al., "An extension of fuzzy decision maps for multi-criteria decision-making”, Egyptian Informatics Journal, 14, No.2, pp.147-155, 2013.

[13] M. Chandrashekhar, A. Shyam Sundar, "Fuzzy Multi-criteria Decision Making associated with Risk and Confidence Attributes", Bulletin of Electrical Engineering and Informatics, Vol. 4, No. 3,pp. 231-240, 2015.

[14] T.Y. Hsieh, et al., "Fuzzy MCDM approach for planning and design tenders selection in public office buildings", International journal of project management, 22, No.7, pp.573-584, 2004.

[15] J.A. Alonso, and M.T. Lamata, “Consistency in the analytic hierarchy process: a new approach", International journal of uncertainty, fuzziness and knowledge-based systems, 14, No.4, pp.445-459, 2006.

[16] J.A. Alonso, and M.T. Lamata, "Estimation of the random index in the analytic hierarchy process", Information processing and management of uncertainty in knowledge-based systems, Vol. 1, pp. 317-322, 2004.

[17] A. Habbal, et al., "Context-aware radio access technology selection in 5G ultra-dense networks", IEEE access, 5, pp.6636-6648, 2017.

[18] E. Roszkowska and D. Kacprzak, "The fuzzy SAW and fuzzy TOPSIS procedures based on ordered fuzzy numbers", Information Sciences, 369, pp.564-584, 2016. 